\title{
Public key exchange using semidirect product of (semi)groups
}

\author{
Maggie Habeeb ${ }^{1}$, Delaram Kahrobaei ${ }^{2}$, Charalambos Koupparis ${ }^{3}$, and \\ Vladimir Shpilrain ${ }^{4}$ \\ 1 California University of Pennsylvania \\ habeeb@calu.edu * \\ ${ }^{2}$ CUNY Graduate Center and City Tech, City University of New York \\ dkahrobaei@gc . cuny.edu *夫 \\ 3 CUNY Graduate Center, City University of New York \\ ckoupparis@gc. cuny.edu \\ 4 The City College of New York and CUNY Graduate Center \\ shpil@groups.sci.ccny.cuny.edu ***
}

\begin{abstract}
In this paper, we describe a brand new key exchange protocol based on a semidirect product of (semi)groups (more specifically, on extension of a (semi)group by automorphisms), and then focus on practical instances of this general idea. Our protocol can be based on any group, in particular on any non-commutative group. One of its special cases is the standard Diffie-Hellman protocol, which is based on a cyclic group. However, when our protocol is used with a non-commutative (semi)group, it acquires several useful features that make it compare favorably to the Diffie-Hellman protocol. Here we also suggest a particular non-commutative semigroup (of matrices) as the platform and show that security of the relevant protocol is based on a quite different assumption compared to that of the standard Diffie-Hellman protocol.
\end{abstract}

\section{Introduction}

It is rare that the beginning of a whole new area of science can be traced back to one particular paper. This is the case with public key cryptography; it started with the seminal paper [2].

The simplest, and original, implementation of the protocol uses the multiplicative group of integers modulo $p$, where $p$ is prime and $g$ is primitive $\bmod p$. A more general description of the protocol uses an arbitrary finite cyclic group.

* Research of Maggie Habeeb was partially supported by the NSF-LSAMP fellowship.

** Research of Delaram Kahrobaei was partially supported by a PSC-CUNY grant from the CUNY research foundation, as well as the City Tech foundation. Research of Delaram Kahrobaei and Vladimir Shpilrain was also supported by the ONR (Office of Naval Research) grant N000141210758.

$\star \star \star$ Research of Vladimir Shpilrain was partially supported by the NSF grants DMS0914778 and CNS-1117675. 
1. Alice and Bob agree on a finite cyclic group $G$ and a generating element $g$ in $G$. We will write the group $G$ multiplicatively.

2. Alice picks a random natural number $a$ and sends $g^{a}$ to Bob.

3. Bob picks a random natural number $b$ and sends $g^{b}$ to Alice.

4. Alice computes $K_{A}=\left(g^{b}\right)^{a}=g^{b a}$.

5. Bob computes $K_{B}=\left(g^{a}\right)^{b}=g^{a b}$.

Since $a b=b a$, both Alice and Bob are now in possession of the same group element $K=K_{A}=K_{B}$ which can serve as the shared secret key.

The protocol is considered secure against eavesdroppers if $G$ and $g$ are chosen properly. The eavesdropper must solve the Diffie-Hellman problem (recover $g^{a b}$ from $g, g^{a}$ and $g^{b}$ ) to obtain the shared secret key. This is currently considered difficult for a "good" choice of parameters (see e.g. [5] for details).

There is an ongoing search for other platforms where the Diffie-Hellman or similar key exchange could be carried out more efficiently, in particular with public/private keys of smaller size. This search already gave rise to several interesting directions, including a whole area of elliptic curve cryptography. We also refer the reader to [6] for a survey of proposed cryptographic primitives based on non-abelian (= non-commutative) groups. A survey of these efforts is outside of the scope of the present paper; our goal here is to suggest a new key exchange protocol based on extension of a (semi)group by automorphisms. Our protocol can be based on any group, in particular on any non-commutative group. It has some superficial resemblance to the classical Diffie-Hellman protocol, but there are several distinctive features that, we believe, give our protocol important advantages. In particular, even though the parties do compute a large power of a public element (as in the classical Diffie-Hellman protocol), they do not transmit the whole result, but rather just part of it.

We also describe in this paper some particular instances of our general protocol. In particular, we suggest a non-commutative semigroup (of matrices) as the platform and show that security of the relevant protocol is based on a quite different assumption compared to that of the standard Diffie-Hellman protocol.

We mention another, rather different, proposal [8] of a cryptosystem based on the semidirect product of two groups and yet another, more complex, proposal of a key agreement based on the semidirect product of two monoids [1. Both these proposals are very different from ours. Also, the extended abstract [3], despite the similarity of the title, has very little overlap with the present paper. In particular, the key exchange protocol in Section 3 of the present paper is brand new.

Finally, we note that the basic construction (semidirect product) we use in this paper can be adopted, with some simple modifications, in other algebraic systems, e.g. associative rings or Lie rings, and key exchange protocols similar to ours can be built on those. 


\section{Semidirect products and extensions by automorphisms}

We include this section to make the exposition more comprehensive. The reader who is uncomfortable with group-theoretic constructions can skip to subsection 2.1

We now recall the definition of a semidirect product:

Definition 1. Let $G, H$ be two groups, let $A u t(G)$ be the group of automorphisms of $G$, and let $\rho: H \rightarrow A u t(G)$ be a homomorphism. Then the semidirect product of $G$ and $H$ is the set

$$
\Gamma=G \rtimes_{\rho} H=\{(g, h): g \in G, h \in H\}
$$

with the group operation given by

$$
(g, h)\left(g^{\prime}, h^{\prime}\right)=\left(g^{\rho\left(h^{\prime}\right)} \cdot g^{\prime}, h \cdot h^{\prime}\right) .
$$

Here $g^{\rho\left(h^{\prime}\right)}$ denotes the image of $g$ under the automorphism $\rho\left(h^{\prime}\right)$, and when we write a product $h \cdot h^{\prime}$ of two morphisms, this means that $h$ is applied first.

In this paper, we focus on a special case of this construction, where the group $H$ is just a subgroup of the group $\operatorname{Aut}(G)$. If $H=\operatorname{Aut}(G)$, then the corresponding semidirect product is called the holomorph of the group $G$. We give some more details about the holomorph in our Section 2.1 and in Section 3 we describe a key exchange protocol that uses (as the platform) an extension of a group $G$ by a cyclic group of automorphisms.

\subsection{Extensions by automorphisms}

A particularly simple special case of the semidirect product construction is where the group $H$ is just a subgroup of the group $\operatorname{Aut}(G)$. If $H=A u t(G)$, then the corresponding semidirect product is called the holomorph of the group $G$. Thus, the holomorph of $G$, usually denoted by $\operatorname{Hol}(G)$, is the set of all pairs $(g, \phi)$, where $g \in G, \phi \in \operatorname{Aut}(G)$, with the group operation given by $(g, \phi) \cdot\left(g^{\prime}, \phi^{\prime}\right)=$ $\left(\phi^{\prime}(g) \cdot g^{\prime}, \phi \cdot \phi^{\prime}\right)$.

It is often more practical to use a subgroup of $\operatorname{Aut}(G)$ in this construction, and this is exactly what we do in Section 3, where we describe a key exchange protocol that uses (as the platform) an extension of a group $G$ by a cyclic group of automorphisms.

Remark 1. One can also use this construction if $G$ is not necessarily a group, but just a semigroup, and/or consider endomorphisms of $G$, not necessarily automorphisms. Then the result will be a semigroup; this is what we use in our Section 6.

\section{$3 \quad$ Key exchange protocol}

In the simplest implementation of the construction described in our Section 2.1. one can use just a cyclic subgroup (or a cyclic subsemigroup) of the group $\operatorname{Aut}(G)$ 
(respectively, of the semigroup $\operatorname{End}(G)$ of endomorphisms) instead of the whole group of automorphisms of $G$.

Thus, let $G$ be a (semi)group. An element $g \in G$ is chosen and made public as well as an arbitrary automorphism $\phi \in A u t(G)$ (or an arbitrary endomorphism $\phi \in \operatorname{End}(G))$. Bob chooses a private $n \in \mathbb{N}$, while Alice chooses a private $m \in \mathbb{N}$. Both Alice and Bob are going to work with elements of the form $\left(g, \phi^{r}\right)$, where $g \in G, r \in \mathbb{N}$. Note that two elements of this form are multiplied as follows: $\left(g, \phi^{r}\right) \cdot\left(h, \phi^{s}\right)=\left(\phi^{s}(g) \cdot h, \phi^{r+s}\right)$.

1. Alice computes $(g, \phi)^{m}=\left(\phi^{m-1}(g) \cdots \phi^{2}(g) \cdot \phi(g) \cdot g, \phi^{m}\right)$ and sends only the first component of this pair to Bob. Thus, she sends to Bob only the element $a=\phi^{m-1}(g) \cdots \phi^{2}(g) \cdot \phi(g) \cdot g$ of the (semi)group $G$.

2. Bob computes $(g, \phi)^{n}=\left(\phi^{n-1}(g) \cdots \phi^{2}(g) \cdot \phi(g) \cdot g, \phi^{n}\right)$ and sends only the first component of this pair to Alice. Thus, he sends to Alice only the element $b=\phi^{n-1}(g) \cdots \phi^{2}(g) \cdot \phi(g) \cdot g$ of the (semi)group $G$.

3. Alice computes $(b, x) \cdot\left(a, \phi^{m}\right)=\left(\phi^{m}(b) \cdot a, x \cdot \phi^{m}\right)$. Her key is now $K_{A}=$ $\phi^{m}(b) \cdot a$. Note that she does not actually "compute" $x \cdot \phi^{m}$ because she does not know the automorphism $x=\phi^{n}$; recall that it was not transmitted to her. But she does not need it to compute $K_{A}$.

4. Bob computes $(a, y) \cdot\left(b, \phi^{n}\right)=\left(\phi^{n}(a) \cdot b, y \cdot \phi^{n}\right)$. His key is now $K_{B}=\phi^{n}(a) \cdot b$. Again, Bob does not actually "compute" $y \cdot \phi^{n}$ because he does not know the automorphism $y=\phi^{m}$.

5. Since $(b, x) \cdot\left(a, \phi^{m}\right)=(a, y) \cdot\left(b, \phi^{n}\right)=(g, \phi)^{m+n}$, we should have $K_{A}=$ $K_{B}=K$, the shared secret key.

Remark 2. Note that, in contrast with the "standard" Diffie-Hellman key exchange, correctness here is based on the equality $h^{m} \cdot h^{n}=h^{n} \cdot h^{m}=h^{m+n}$ rather than on the equality $\left(h^{m}\right)^{n}=\left(h^{n}\right)^{m}=h^{m n}$. In the "standard" DiffieHellman set up, our trick would not work because, if the shared key $K$ was just the product of two openly transmitted elements, then anybody, including the eavesdropper, could compute $K$.

\section{Computational cost}

From the look of transmitted elements in our protocol in Section 3, it may seem that the parties have to compute a product of $m$ (respectively, $n$ ) elements of the (semi)group $G$. However, since the parties actually compute powers of an element of $G$, they can use the "square-and-multiply" method, as in the standard Diffie-Hellman protocol. Then there is a cost of applying an automorphism $\phi$ to an element of $G$, and also of computing powers of $\phi$. These costs depend, of course, on a specific platform (semi)group that is used with our protocol. In our first, "toy" example (Section 5 below), both applying an automorphism $\phi$ and computing its powers amount to exponentiation of elements of $G$, which can 
be done again by the "square-and-multiply" method. In our main example, in Section [6, $\phi$ is a conjugation, so applying $\phi$ amounts to just two multiplications of elements in $G$, while computing powers of $\phi$ amounts to exponentiation of two elements of $G$ (namely, of the conjugating element and of its inverse).

Thus, in either instantiation of our protocol considered in this paper, the cost of computing $(g, \phi)^{n}$ is $O(\log n)$, just as in the standard Diffie-Hellman protocol.

\section{5 "Toy example": multiplicative $\mathbb{Z}_{p}^{*}$}

As one of the simplest instantiations of our protocol, we use here the multiplicative group $\mathbb{Z}_{p}^{*}$ as the platform group $G$ to illustrate what is going on. In selecting a prime $p$, as well as private exponents $m, n$, one can follow the same guidelines as in the "standard" Diffie-Hellman.

Selecting the (public) endomorphism $\phi$ of the group $\mathbb{Z}_{p}^{*}$ amounts to selecting yet another integer $k$, so that for every $h \in \mathbb{Z}_{p}^{*}$, one has $\phi(h)=h^{k}$. If $k$ is relatively prime to $p-1$, then $\phi$ is actually an automorphism. Below we assume that $k>1$.

Then, for an element $g \in \mathbb{Z}_{p}^{*}$, we have:

$$
(g, \phi)^{m}=\left(\phi^{m-1}(g) \cdots \phi(g) \cdot \phi^{2}(g) \cdot g, \phi^{m}\right) .
$$

We focus on the first component of the element on the right; easy computation shows that it is equal to $g^{k^{m-1}+\ldots+k+1}=g^{\frac{k^{m}-1}{k-1}}$. Thus, if the adversary chooses a "direct" attack, by trying to recover the private exponent $m$, he will have to solve the discrete log problem twice: first to recover $\frac{k^{m}-1}{k-1}$ from $g^{\frac{k^{m}-1}{k-1}}$, and then to recover $m$ from $k^{m}$. (Note that $k$ is public since $\phi$ is public.)

On the other hand, the analog of what is called "the Diffie-Hellman problem" would be to recover the shared key $K=g^{\frac{k^{m+n}-1}{k-1}}$ from the triple $\left(g, g^{\frac{k^{m}-1}{k-1}}, g^{\frac{k^{n}-1}{k-1}}\right)$. Since $g$ and $k$ are public, this is equivalent to recovering $g^{k^{m+n}}$ from the triple $\left(g, g^{k^{m}}, g^{k^{n}}\right)$, i.e., this is exactly the standard Diffie-Hellman problem.

Thus, the bottom line of this example is that the instantiation of our protocol where the group $G$ is $\mathbb{Z}_{p}^{*}$, is not really different from the standard Diffie-Hellman protocol. In the next section, we describe a more interesting instantiation, where the (semi)group $G$ is non-commutative.

\section{Matrices over group rings and extensions by inner automorphisms}

To begin with, we note that our general protocol in Section 3 can be used with any non-commutative group $G$ if $\phi$ is selected to be a non-trivial inner automorphism, i.e., conjugation by an element which is not in the center of $G$. Furthermore, it can be used with any non-commutative semigroup $G$ as well, as long as $G$ has some invertible elements; these can be used to produce inner 
automorphisms. A typical example of such a semigroup would be a semigroup of matrices over some ring.

In the paper [4, the authors have employed matrices over group rings of a (small) symmetric group as platforms for the (standard) Diffie-Hellman-like key exchange. In this section, we use these matrix semigroups again and consider an extension of such a semigroup by an inner automorphism to get a platform semigroup for our protocol.

Recall that a (semi)group ring $R[S]$ of a (semi)group $S$ over a commutative $\operatorname{ring} R$ is the set of all formal sums

$$
\sum_{g_{i} \in S} r_{i} g_{i}
$$

where $r_{i} \in R$, and all but a finite number of $r_{i}$ are zero.

The sum of two elements in $R[G]$ is defined by

$$
\left(\sum_{g_{i} \in S} a_{i} g_{i}\right)+\left(\sum_{g_{i} \in S} b_{i} g_{i}\right)=\sum_{g_{i} \in S}\left(a_{i}+b_{i}\right) g_{i} .
$$

The multiplication of two elements in $R[G]$ is defined by using distributivity. As we have already pointed out, if a (semi)group $G$ is non-commutative and has non-central invertible elements, then it always has a non-identical inner automorphism, i.e., conjugation by an element $g \in G$ such that $g^{-1} h g \neq h$ for at least some $h \in G$.

Now let $G$ be the semigroup of $3 \times 3$ matrices over the group ring $\mathbb{Z}_{7}\left[A_{5}\right]$, where $A_{5}$ is the alternating group on 5 elements. Here we use an extension of the semigroup $G$ by an inner automorphism $\varphi_{H}$, which is conjugation by a matrix $H \in G L_{3}\left(\mathbb{Z}_{7}\left[A_{5}\right]\right)$. Thus, for any matrix $M \in G$ and for any integer $k \geq 1$, we have

$$
\varphi_{H}(M)=H^{-1} M H ; \varphi_{H}^{k}(M)=H^{-k} M H^{k} .
$$

Now our general protocol from Section 3 is specialized in this case as follows.

1. Alice and Bob agree on public matrices $M \in G$ and $H \in G L_{3}\left(\mathbb{Z}_{7}\left[A_{5}\right]\right)$. Alice selects a private positive integer $m$, and Bob selects a private positive integer $n$.

2. Alice computes $\left(M, \varphi_{H}\right)^{m}=\left(H^{-m+1} M H^{m-1} \cdots H^{-2} M H^{2} \cdot H^{-1} M H \cdot M, \varphi_{H}^{m}\right)$ and sends only the first component of this pair to Bob. Thus, she sends to Bob only the matrix

$$
A=H^{-m+1} M H^{m-1} \cdots H^{-2} M H^{2} \cdot H^{-1} M H \cdot M=H^{-m}(H M)^{m} .
$$


3. Bob computes $\left(M, \varphi_{H}\right)^{n}=\left(H^{-n+1} M H^{n-1} \cdots H^{-2} M H^{2} \cdot H^{-1} M H \cdot M, \varphi_{H}^{n}\right)$ and sends only the first component of this pair to Alice. Thus, he sends to Alice only the matrix

$$
B=H^{-n+1} M H^{n-1} \cdots H^{-2} M H^{2} \cdot H^{-1} M H \cdot M=H^{-n}(H M)^{n} .
$$

4. Alice computes $(B, x) \cdot\left(A, \varphi_{H}^{m}\right)=\left(\varphi_{H}^{m}(B) \cdot A, x \cdot \varphi_{H}^{m}\right)$. Her key is now $K_{\text {Alice }}=\varphi_{H}^{m}(B) \cdot A=H^{-(m+n)}(H M)^{m+n}$. Note that she does not actually "compute" $x \cdot \varphi_{H}^{m}$ because she does not know the automorphism $x=\varphi_{H}^{n}$; recall that it was not transmitted to her. But she does not need it to compute $K_{\text {Alice }}$.

5. Bob computes $(A, y) \cdot\left(B, \varphi_{H}^{n}\right)=\left(\varphi_{H}^{n}(A) \cdot B, y \cdot \varphi_{H}^{n}\right)$. His key is now $K_{B o b}=$ $\varphi_{H}^{n}(A) \cdot B$. Again, Bob does not actually "compute" $y \cdot \varphi_{H}^{n}$ because he does not know the automorphism $y=\varphi_{H}^{m}$.

6. Since $(B, x) \cdot\left(A, \varphi_{H}^{m}\right)=(A, y) \cdot\left(B, \varphi_{H}^{n}\right)=\left(M, \varphi_{H}\right)^{m+n}$, we should have $K_{\text {Alice }}=K_{B o b}=K$, the shared secret key.

\section{Security assumptions and analysis}

In this section, we address the question of security of the particular instantiation of our protocol described in Section 6 .

Recall that the shared secret key in the protocol of Section 6 is

$$
K=\varphi_{H}^{m}(B) \cdot A=\varphi_{H}^{n}(A) \cdot B=H^{-(m+n)}(H M)^{m+n} .
$$

Therefore, our security assumption here is that it is computationally hard to retrieve the key $K=H^{-(m+n)}(H M)^{m+n}$ from the quadruple

$\left(H, M, H^{-m}(H M)^{m}, H^{-n}(H M)^{n}\right)$.

In particular, we have to take care that the matrices $H$ and $H M$ do not commute because otherwise, $K$ is just a product of $H^{-m}(H M)^{m}$ and $H^{-n}(H M)^{n}$.

A weaker security assumption arises if an eavesdropper tries to recover a private exponent from a transmission, i.e., to recover, say, $m$ from $H^{-m}(H M)^{m}$. A special case of this problem, where $H=I$, is the "discrete log" problem for matrices over $\mathbb{Z}_{7}\left[A_{5}\right]$, namely: recover $m$ from $M$ and $M^{m}$. Even this problem appears to be hard; it was addressed in [4 in more detail. In particular, statistical experiments show that for a random matrix $M$, matrices $M^{m}$ are indistinguishable from random.

In order to verify the robustness and security of our protocol, we have experimentally addressed two questions. The first question is whether or not any information about the private exponent $n$ is leaked from transmission. That is, for a random exponent $n$, how different is the matrix $\left(M, \varphi_{H}\right)^{n}$ from $N$, where $\mathrm{N}$ is random? The second point that needs verification is to determine how different the final shared key is from a random matrix. More specifically, if Alice and Bob choose secret integers $m$ and $n$ respectively, how different is the matrix $\left(M, \varphi_{H}\right)^{n+m}$ from $\left(M, \varphi_{H}\right)^{q}$, where $q$ is of the same bit size are $n+m$. 
To perform the first experimental validation we worked over $M_{3}\left(\mathbb{Z}_{7}\left[A_{5}\right]\right)$ and used random choices of $n \in\left[10^{44}, 10^{55}\right]$. We then looked at the two distributions generated by the first component of $\left(M, \varphi_{H}\right)^{n}$ and $N$, where $M$ and $N$ are random matrices. We need to verify that the two generated distributions are in fact indistinguishable. To this end we looked at the components of each matrix and counted the frequency of occurrence of each element of $A_{5}$. We repeated this process 500 times and generated a frequency distribution table for the two distributions.

From the table, we produced $Q-Q$ (quantile) plots of the entries of the two matrices: the first component of $\left(M, \varphi_{H}\right)^{n}$ and a random matrix $N$. Quantile plots are a quick graphical tool for comparing two distributions. These plots essentially compare the cumulative distribution functions of two distributions. If the distributions are identical, the resulting graph will be a straight line.

QQ Plot of frequency of elements of $A_{5}$ occuring in entries of $M^{n} v s ~ N$
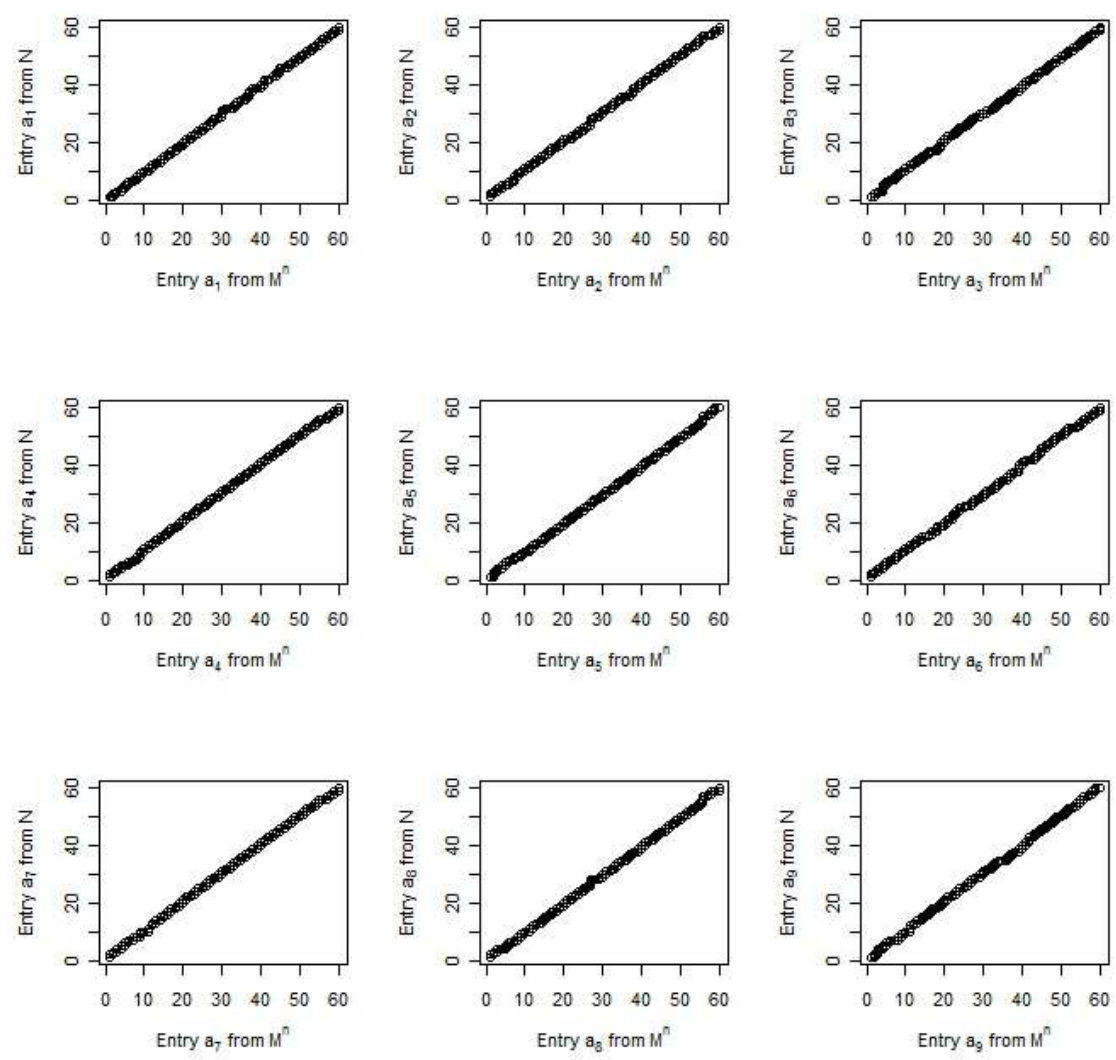

Fig. 1. Results for $M^{n}$ vs. $N$ 
Figure 1 shows the resulting plots for this experiment. These graphs show that the two distributions are in fact identical, therefore suggesting that no information about a private exponent $n$ is revealed by transmissions between Alice and Bob.

The second experiment we carried out was similar to the first one, except in this case we were comparing the first components of $\left(M, \varphi_{H}\right)^{n}$ and $\left(M, \varphi_{H}\right)^{a+b}$, where $n, a$ and $b$ are random and all of roughly the same bit size, i.e. all are integers from $\left[10^{44}, 10^{55}\right]$. This experiment helps address the DDH (decisional Diffie-Hellman) assumption by comparing the shared secret key to a random key and ensuring that no information about the former is leaked. See Figure 2 for the resulting $Q-Q$ plots. These 9 graphs suggest that the two distributions generated by these keys are in fact indistinguishable.

QQ Plot of frequency of elements of $A_{5}$ occuring in entries of $M^{n}$ vs $M^{a+b}$
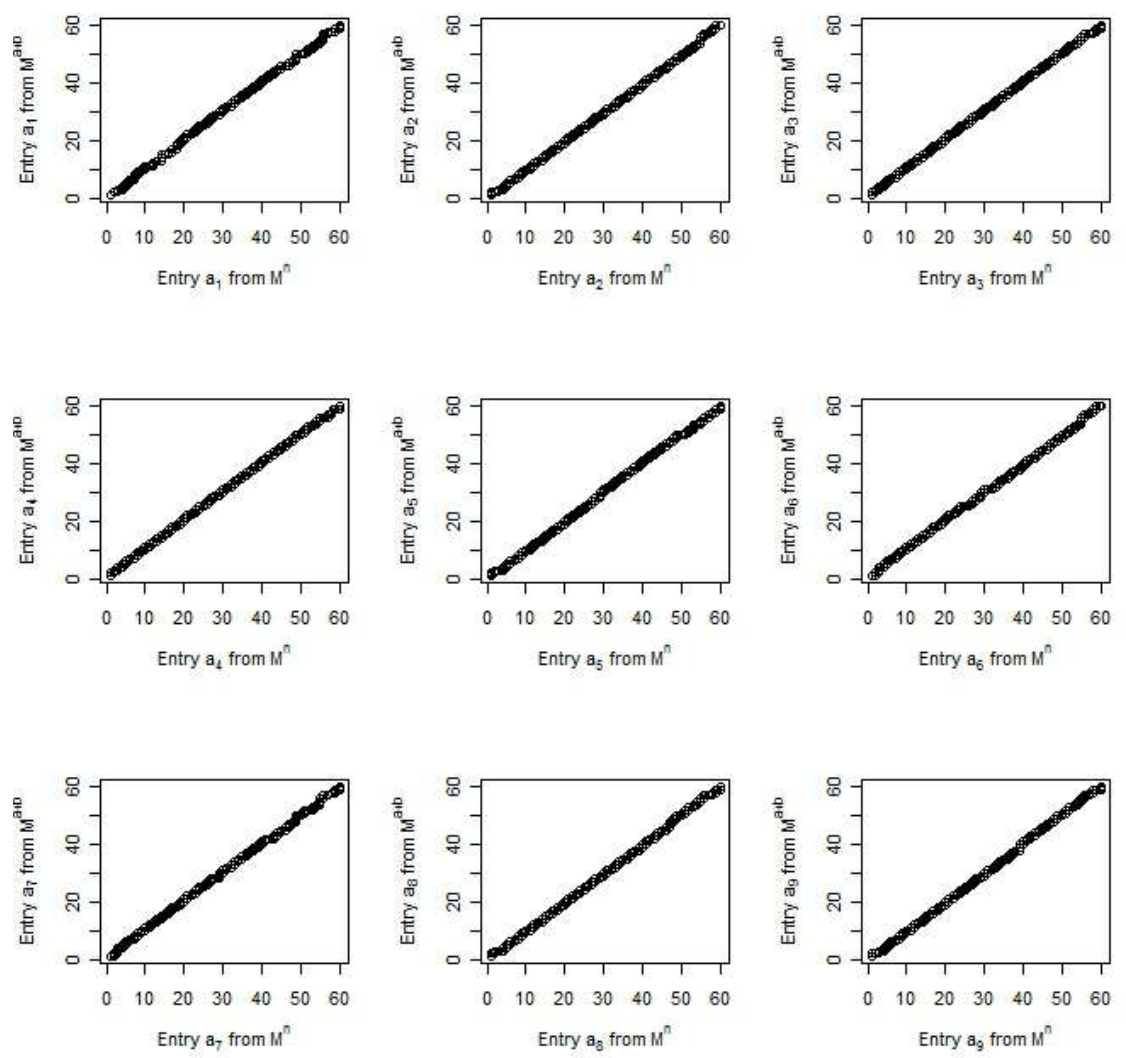

Fig. 2. Results for $M^{n}$ vs. $M^{a+b}$ 


\section{Parameters and key generation}

Private exponents $m$ and $n$ should be of the magnitude of $2^{t}$, where $t$ is the security parameter, to make brute force search infeasible. Thus, $m$ and $n$ are roughly $t$ bits long.

Public matrix $M$ is selected as a random $3 \times 3$ matrix over the group ring $\mathbb{Z}_{7}\left[A_{5}\right]$, which means that each entry of $M$ is a random element of $\mathbb{Z}_{7}\left[A_{5}\right]$. The latter means that each entry is a sum $\sum_{g_{i} \in A_{5}} c_{i} g_{i}$ of elements of the group $A_{5}$ with coefficients $c_{i}$ selected uniformly randomly from $\mathbb{Z}_{7}$. Thus, although the bit complexity of the matrix $M$ is fairly high $(9 \cdot 3 \cdot 60=1620$ bits $)$, the procedure for sampling $M$ is quite efficient. We want to impose one restriction on the matrix $M$ though. There is a trivialization (sometimes called augmentation) homomorphism of the group ring that sends every group element to 1 . This homomorphism naturally extends to a homomorphism of the whole semigroup of matrices. To avoid leaking any information upon applying this homomorphism, we want the image of every entry of $M$ to be 0 . Group ring elements like that are easy to sample: after sampling a random element $\sum_{g_{i} \in A_{5}} c_{i} g_{i}$ of $\mathbb{Z}_{7}\left[A_{5}\right]$, we select a random coefficient $c_{i}$ and change it, if necessary, to have $\sum_{i} c_{i}=0$.

Note that with this choice of $M$, applying the trivialization homomorphism to any of the transmitted matrices in our protocol will produce the zero matrix, thus not leaking any information. We also note that there are no other homomorphisms of the group $A_{5}$ (which is a finite simple group), except for inner automorphisms. This will prevent an eavesdropper from learning partial information about secret keys by applying homomorphisms to transmitted matrices.

Finally, we need to sample an invertible $3 \times 3$ matrix $H$ over the group ring $\mathbb{Z}_{7}\left[A_{5}\right]$. There are several techniques for doing this; here we give a brief exposition of one possible procedure.

We start with an already "somewhat random" matrix, for which it is easy to compute the inverse. An example of such a matrix is a lower/upper triangular matrix, with invertible elements on the diagonal:

$$
U=\left(\begin{array}{ccc}
g_{1} & u_{1} & u_{2} \\
0 & g_{2} & u_{3} \\
0 & 0 & g_{3}
\end{array}\right) .
$$

Here $g_{i}$ are random elements of the group $A_{5}$, and $u_{i}$ are random elements of the group ring $\mathbb{Z}_{7}\left[A_{5}\right]$. We then take a random product, with 20 factors, of such random invertible upper and lower triangular matrices, to get our invertible matrix $H$.

We note that there is always a concern (also in the standard Diffie-Hellman protocol) about the order of a public element: if the order is too small, then a brute force attack may be feasible. In our situation, this concern is significantly alleviated by the fact that our transmissions are products of powers of two different matrices rather than powers of a single matrix. Therefore, even if the order of one of the matrices happens to be small by accident, this does not mean that the product $H^{-m}(H M)^{m}$ will go into loop of a small size. Furthermore, 
since our matrix $M$ is non-invertible, it does not have an "order", but rather a loop: $M^{r}=M^{s}$ for some positive $r \neq s$. The matrices $H M$ and $H^{-m}(H M)^{m}$ are non-invertible, too, so they do not have an order either, but rather a loop. Detecting a loop is, in general, computationally much harder than computing the order of an invertible element.

\section{Conclusions}

We have presented a brand new key exchange protocol based on extension of a (semi)group by automorphisms and described some practical instances of this general idea. Our protocol can be based on any group, in particular on any noncommutative group. It has some superficial resemblance to the classical DiffieHellman protocol, but there are several distinctive features that, we believe, give our protocol important advantages:

- Even though the parties do compute a large power of a public element (as in the classical Diffie-Hellman protocol), they do not transmit the whole result, but rather just part of it.

- Since the classical Diffie-Hellman protocol is a special case of our protocol, breaking our protocol even for any cyclic group would imply breaking the DiffieHellman protocol.

- If the platform (semi)group is not commutative, then we get a new security assumption. In the simplest case, where the automorphism used for extension is inner, attacking a private exponent amounts to recovering an integer $n$ from a product $g^{-n} h^{n}$, where $g, h$ are public elements of the platform (semi)group. In the special case where $g=1$ this boils down to recovering $n$ from $h^{n}$, with public $h$ ("discrete log" problem).

On the other hand, in the particular instantiation of our protocol, which is based on a non-commutative semigroup extended by an inner automorphism, recovering the shared secret key from public information is based on a different security assumption than the classical Diffie-Hellman protocol is. Namely, the assumption is that it is computationally hard to retrieve the shared secret key $K=h^{-(m+n)} g^{m+n}$ from the triple of elements $\left(h, h^{-m} g^{m}, h^{-n} g^{n}\right)$, assuming that $g$ and $h$ do not commute.

\section{References}

1. I. Anshel, M. Anshel, D. Goldfeld, and S. Lemieux, Key agreement, the Algebraic Eraser, and lightweight cryptography, Algebraic methods in cryptography, Contemp. Math. Amer. Math. Soc. 418 (2006), 1-34.

2. W. Diffie and M. E. Hellman, New Directions in Cryptography, IEEE Transactions on Information Theory IT-22 (1976), 644-654.

3. M. Habeeb, D. Kahrobaei and V. Shpilrain, A public key exchange using semidirect products of groups (extended abstract), Proceedings of the International Conference in Symbolic Computations and Cryptography, SCC 2010, Royal Holloway, University of London, Egham, United Kingdom, June 2010. 
4. D. Kahrobaei, C. Koupparis, V. Shpilrain, Public key exchange using matrices over group rings, Groups, Complexity, and Cryptology, to appear. http://arxiv.org/abs/1302.1625

5. A. Menezes, P. van Oorschot, and S. Vanstone, Handbook of Applied Cryptography, CRC-Press 1996.

6. A. G. Myasnikov, V. Shpilrain, and A. Ushakov, Group-based cryptography, Birkhäuser 2008.

7. A. G. Myasnikov, V. Shpilrain, and A. Ushakov, Non-commutative cryptography and complexity of group-theoretic problems, Amer. Math. Soc. Surveys and Monographs, 2011.

8. S.-H. Paeng, K.-C. Ha, J. H. Kim, S. Chee and C. Park, New public key cryptosystem using finite non-abelian groups, in: Crypto 2001, Lecture Notes Comp. Sc. 2139 (2001), 470-485. 